AperTO - Archivio Istituzionale Open Access dell'Università di Torino

\title{
Autozygosity-driven genetic diagnosis in consanguineous families from Italy and the Greater Middle East
}

\section{This is the author's manuscript}

Original Citation:

\section{Availability:}

This version is available http://hdl.handle.net/2318/1740332

since 2020-10-23T12:36:15Z

Published version:

DOI:10.1007/s00439-020-02187-7

Terms of use:

Open Access

Anyone can freely access the full text of works made available as "Open Access". Works made available under a Creative Commons license can be used according to the terms and conditions of said license. Use of all other works requires consent of the right holder (author or publisher) if not exempted from copyright protection by the applicable law. 
Autozygosity-driven genetic diagnosis in consanguineous families from Italy and the Greater Middle East

Flavia Palombo ${ }^{1,2}$, Claudio Graziano ${ }^{1}$, Nadia Al Wardy ${ }^{3}$, Nayereh Nouri ${ }^{4,5}$, Caterina Marconi ${ }^{6}$, Pamela Magini ${ }^{1}$, Giulia Severi ${ }^{1}$, Chiara La Morgia ${ }^{2,7}$, Gaetano Cantalupo ${ }^{8,9}$, Duccio Maria Cordelli $^{6,10}$, Simone Gangarossa ${ }^{11}$, Mohammed Nasser Al Kindi ${ }^{3}$, Mazin Al Khabouri ${ }^{3,12}$, Mansoor Salehi $^{4}$, Elisa Giorgio ${ }^{13}$, Alfredo Brusco ${ }^{13}$, Francesco Pisani ${ }^{14}$, Giovanni Romeo ${ }^{6}$, Valerio Carelli ${ }^{2,7}$, Tommaso Pippucci ${ }^{1} \S$, Marco Seri ${ }^{1,6}$

1 Medical Genetics Sant’Orsola-Malpighi University Hospital of Bologna, Italy

2 IRCCS Istituto delle Scienze Neurologiche di Bologna, UOC Clinica Neurologica, Bologna, Italy

3 Department of Biochemistry, College of Medicine and Health Sciences, Sultan Qaboos University, Muscat, Oman

4 Department of Genetics and Molecular Biology, Isfahan University of Medical Sciences, Isfahan, Iran.

5 Craniofacial and Cleft Research Center, Isfahan University of Medical Sciences, Isfahan, Iran 6 Department of Medical and Surgical Sciences (DIMEC), University of Bologna, Bologna, Italy 7 Department of Biomedical and Neuromotor Sciences (DIBINEM), University of Bologna, Bologna, Italy

8 Child Neuropsychiatry, Department of Surgical Sciences, Dentistry, Gynecology and Pediatrics, University of Verona, Verona, Italy 
9 UOC Neuropsichiatria Infantile, DAI Materno-Infantile, AOUI Verona, Verona, Italy

10 Neuropsychiatry Sant’Orsola-Malpighi University Hospital of Bologna, Bologna, Italy

11 ASP7 Ragusa, Ragusa, Italy

12 Department of ENT, Al Nahdha Hospital, Ministry of Health, Muscat, Oman

13 Department of Medical Sciences, University of Torino, Turin, Italy

14 Child Neuropsychiatry Unit, Department of Medicine \& Surgery, University of Parma, Parma, Italy

$\S$ Correspondence to Tommaso Pippucci Via Massarenti 940138 Bologna Italy Tel 00390512088421

Mail 


\section{Abstract}

Autozygosity-driven exome analysis has been shown effective for identification of genes underlying recessive diseases especially in countries of the so-called Greater Middle East (GME), where high consanguinity unravels the phenotypic effects of recessive alleles and large family sizes facilitate homozygosity mapping. In Italy, as in most European countries, consanguinity is estimated low. Nonetheless, consanguineous Italian families are not uncommon in publications of genetic findings and are often key to new associations of genes with rare diseases. We collected 52 patients from 47 consanguineous families with suspected recessive diseases, 29 originated in GME countries and 18 of Italian descent. We performed autozygosity-driven exome analysis by detecting long runs of homozygosity (ROHs>1.5 Mb) and by prioritizing candidate clinical variants within. We identified a pathogenic synonymous variant that had been previously missed in NARS2 and we increased an initial high diagnostic rate (47\%) to $55 \%$ by matchmaking our candidate genes and including in the analysis shorter ROHs that may also happen to be autozygous. GME and Italian families contributed to diagnostic yield comparably. We found no significant difference either in the extension of the autozygous genome, or in the distribution of candidate clinical variants between GME and Italian families, while we showed that the average autozygous genome was larger and the mean number of candidate clinical variants was significantly higher $(\mathrm{p}=0.003)$ in mutation-positive than in mutationnegative individuals, suggesting that these features influence the likelihood that the disease is autozygosity-related. We highlight the utility of autozygosity-driven genomic analysis also in countries and/or communities where consanguinity is not widespread cultural tradition. 


\section{Introduction}

In genetics, consanguineous marriages are commonly defined as unions between individuals related as second cousins or closer, resulting in a pedigree-based coefficient of inbreeding (F) in their progeny $\geq 0.0156$ (Bittles, 2001). Individuals whose parents are so closely related are expected to have an increased proportion of their autosomal genome that is autozygous, where two identical haplotypes descend from a recent common ancestor; the closer the degree of relatedness, the greater the proportion of the genome that is expected to be autozygous (Smith, 1974). Autozygosity is considered to be the genomic hallmark of inbreeding, manifesting as long runs of homozygosity (ROHs), i.e. sizeable stretches of homozygous genotypes at consecutive polymorphic DNA marker positions, which are spread throughout an individual genome.

The most well-known medical impact of parental consanguinity is the increased risk of rare autosomal recessive diseases in the progeny. Notably, the excess risk is inversely proportional to the frequency of the disease-related allele in the gene pool (Bittles, 2001). As a result, genetic analysis of consanguineous families paved the way to the identification of many genes underlying ultra-rare Mendelian conditions (Alkuraya, 2013; Alazami et al., 2015). Since it was first proposed in 1987 (Lander and Botstein 1987) homozygosity mapping, which exploits the occurrence of long ROHs surrounding the disease-related variant, proved to be a powerful tool to map recessive genes, and its combination with Whole Exome Sequencing (WES) rapidly boosted novel gene discoveries in first years of the next generation sequencing era (Aldahmesh et al., 2011; Becker et al.,2011; Chiang et al., 2012; Shaheen al., 2011; Sobreira et al., 2010). More recently, diagnostic yield up to 60\% has been reported in clinical settings where homozygosity mapping and WES have been used to establish genetic diagnosis in children to consanguineous parents (Makrythanasis et al., 2014; Yavarna et al., 2015; Charng et al., 2016). It is usually emphasized how marriage between consanguineous spouses is a cultural tradition in countries of the so-called Greater Middle East (GME) (Scott et al., 2016) while is no longer common among most European populations where, however, it was socially 
accepted and even favored at least until the 19th century (Bittles et al., 2010). In Italy, profound demographic changes occurred during the 2nd half of the 20th century causing a rapid decrease in rates of consanguineous marriages, especially in the north (Cavalli Sforza et al., 2004). It has been argued that autozygosity-driven WES analysis is most powerful in populations of GME countries where the identification of genes underlying autosomal recessive diseases is facilitated by high population inbreeding rate and large family size (Monies et al., 2017). Nonetheless, reports of genetic diagnoses and novel disease-gene associations in Italian consanguineous families are not uncommon in the literature (see Spataro et al., 2019; Ramos et al., 2019; Milev et al., 2018 as illustrative recent papers). We aimed to investigate whether autozygosity-driven WES analysis could be as effective in a country like Italy where population inbreeding rate is lower and family size is on average smaller than in GME countries. Here we report on our findings in 52 patients from 47 consanguineous families with suspected autosomal recessive diseases, 29 that originated in GME countries and 18 of Italian descent. 


\section{Materials and Methods}

Patients. This study includes 47 families referred in years 2012-2017 to the Medical Genetics Unit, Sant'Orsola-Malpighi University Hospital in Bologna, Italy or collaborative centers (Bellaria Hospital in Bologna, Italy; Città della Salute e della Scienza University Hospital, Turin, Italy; Department of Biochemistry, College of Medicine and Health Sciences, Sultan Qaboos University, Muscat, Oman; Department of ENT, Al Nahdha Hospital, Ministry of Health, Muscat, Oman; Alzahra University Hospital, Isfahan University of Medical Sciences, Isfahan, Iran) for suspected genetic condition in consanguineous families.

Written informed consent was obtained from all subjects and study was approved by the local institution ethical committee and review board (IRB protocol 3206/2016 at Policlinico S. OrsolaMalpighi, Bologna (Italy); Ref No. SQU - EC/121/16, MREC \# 1311 (Oman); Ethics Committee of the Città della Salute e della Scienza University Hospital, Torino (Italy) (approval number 0060884); IRM. MUI. REC of Isfahan University of Medical science (Iran)) and performed according to the Declaration of Helsinki protocol.

Whole Exome Sequencing. Genomic DNA was extracted from peripheral blood samples collected in EDTA anticoagulant with the GenElute Blood Genomic DNA Kit (Sigma Aldrich, Missouri, USA), following the manufacturer’s instruction, and with the QIAamp DNA Blood Mini (Qiagen, Venlo, Netherlands), following a modified protocol (800 $\mu$ l of blood instead of $200 \mu \mathrm{l}$ and 2 additional washes with the kit's buffer) to obtain $3 \mu \mathrm{g}$ of DNA.

Targeted capture and enrichment were performed using different commercial kits: BGI exome (BGI Tech Solutions, Shenzhen, China), Nextera Rapid CaptureExome (Illumina Inc., San Diego, CA), TruSeqExomeLibrary Prep Kit (Illumina), SeqCap EZ Exome Enrichment v2 and v3 (Nimblegen Inc., Madison, WI). Libraries were sequenced as 91-bp or 100-bp paired-end reads on Illumina HiSeq2000 or HiSeq2500 platforms (Illumina). 
Generated reads were treated following a general pipeline elsewhere described (Magini et al., HMG 2014) including alignment with BWA ( $\mathrm{Li} \mathrm{H}$ and Durbin R, 2010) to the reference genome hg19, realignment and base quality score recalibration with GATK (DePristo MA et al., 2011) and duplicate removal with PicardTools (http://picartools.sourceforge.net). Alignment and coverage statistics were collected with SAMtools (Li H et al., 2009) and GATK. Variants were called and filtered by quality with GATK HaplotypeCaller and Variant Quality Score Recalibration, and then annotated with RefSeq using ANNOVAR (Wang K et al., 2010). H3M2 (Magi et al., 2014) was used for the identification of ROHs from WES alignments.

Autozygosity-based variant prioritization workflow. To prioritize variants according to their probability of being causal for autozygosity-related pathology, we classified ROHs identified by H3M2 into 2 size classes reflecting the presumed ROH origin (Pemberton et al., 2012):

1. long $\mathrm{ROH}$ (larger than $1.5 \mathrm{Mb}$ ), typical of consanguineous families as a consequence of close parental relatedness and thus likely to be autozygous

2. short-medium $\mathrm{ROH}$ (smaller than $1.5 \mathrm{Mb}$ ), common in inbred communities but also present in outbred populations as a consequence of background parental relatedness;

Candidate clinical variants were defined as variants with potential to alter the protein product (missense, nonsense, small insertion/deletions and splicing-affecting variants) with allele frequency lower than 0.01 (rare variants from here on) and not seen in homozygous state in gnomAD database (https://gnomad.broadinstitute.org/). Effect on splicing was assumed if variants impacted conventional splice-sites ( $\pm 2 \mathrm{bp}$ from intron-exon junction) and/or were predicted as unconventional splicing-affecting variants (uSAVs) by MutPredSplice v1.3.2 (http://mutdb.org/mutpredsplice).

We subsequently stratified homozygous candidate clinical variants following their probability of being autozygous: autosomal homozygous variants in long ROHs (increased chance to be autozygous) and out of long ROHs (less likely to be autozygous). 
Finally, we prioritized candidate clinical variants in 3 layers. Based on the American College for Medical Genetics (ACMG) (Richards et al., 2015) guidelines the first two layers were:

a) pathogenic:

- variant already reported as pathogenic or likely pathogenic in ClinVar (https://www.ncbi.nlm.nih.gov/clinvar/) and/or $\quad$ in $\quad$ HGMD® (http://www.hgmd.cf.ac.uk/ac/index.php) or for which pathogenicity is convincingly supported by data in the literature;

- $\quad$ variant predicted to be Loss of Function (LOF) (nonsense changes, frame-shift indels and splicing-affecting variants) within a gene reported in Online Mendelian Inheritance in Man (OMIM) to be associated with a Mendelian phenotype;

b) likely pathogenic:

- non-LOF variant within a gene reported in OMIM to be associated with a Mendelian phenotype, consistent with the clinical diagnosis;

The third layer included potentially pathogenic variants predicted to be deleterious by Combined Annotation Dependent Depletion (CADD v.1.3) score greater than 20 and in genes not reported in OMIM to be associated with a Mendelian phenotype at the time when bioinformatic analysis was completed (31/12/2017).

Sanger Sequencing. Variants were validated and their segregation within parents and siblings was checked through Sanger sequencing. Specific primers (Eurofins Genomics) were manually designed and PCRs were carried out using KAPA ReadyMix (KAPA Biosystems) under standard condition. Sanger sequencing was performed using the Big Dye Terminator v1.1 Cycle Sequencing Kit (Applied Biosystems), following the manufacturer's instructions. Sequencing analysis was performed by the automated sequencer with 48 capillaries (3730 DNA analyzer, Applied Biosystems) and output files were analyzed through the software Sequencher 5.0 (Gene Code Corporation). 
NARS2 cDNA sequencing and Real time PCR. Total RNA was extracted from cultured skeletal muscle cells and fibroblasts of a healthy individual and the proband of family ITA_9, by using the tissue protocol of QIAmp RNA blood mini kit (Qiagen). 500 ng of extracted RNA and of human colon and whole brain RNA pools (Clontech) were retrotranscribed to cDNA through the highcapacity cDNA reverse transcription kit (Thermo Fisher Scientific). To evaluate possible splicing alterations around exon 9 in the patient, NARS2 cDNA was specifically amplified through standard PCR with primers complementary to exon 7 (forward) and exon 10 (reverse) sequences. Bands from gel electrophoresis of NARS2 cDNA amplicons were separated, purified through the QIAquick gel extraction kit (Qiagen), sequenced by using the BigDye terminator v1.1 cycle sequencing kit (Thermo Fisher Scientific) and run on the 3730 DNA Analyzer (Thermo Fisher Scientific). Expression of NARS2 wt transcript, including exon 9, was evaluated in patient's and controls' fibroblasts and skeletal muscle cells by quantitative PCR through the Universal Probe Library (UPL) system (Roche). ACTB mRNA was used as endogenous normalizer and amplified separately. Each reaction was performed in triplicate. The $\Delta \mathrm{Ct}$ method was applied to real-time data to obtain a relative quantification of NARS2 wt transcript expression.

Statistical analysis. Differences between mean values of coverage, proportion of autozygous genome and number of variants were assessed by Welch's t-test, or unequal variances t-test, with $\mathrm{R}$ version 3.5.1. (https://www.r-project.org/). A p-value $\leq 0.05$ was considered significant. 


\section{Results}

Patients' population and autozygosity-driven WES variant prioritization. In this study, 52 individuals affected with different disorders of suspected genetic origin, belonging to 47 consanguineous families, had WES performed. In detail, parents’ probands were related as first cousins (40, 85.1\%), first cousins once-removed (3, 6.4\%), second cousins (3, 6.4\%) or second cousins once-removed (1, 2.1\%). Family history was negative for 43 probands (91.5\%), while the remaining had 1 (3 families, $6.5 \%$ ) or 2 (1 family, 2\%) affected siblings but no record of disease was reported for parents or relatives in upper generations, suggesting autosomal recessive inheritance. The majority of families (29, 62\%) were from GME countries, while the remaining (18, 38\%) were from Italy. There was no major difference in the distribution of the degree of parental relatedness between Italian and GME families, with first cousins being preponderant in both (13/18 [72\%] and 27/29 [93\%], respectively). Clinical diagnoses reflected a composition of suspected Mendelian disorders, including either groups of families from single GME countries with homogeneous phenotype (12 Omani families with nonsyndromic congenital deafness) or with syndromic conditions unified by a common sign (8 Iranian families with syndromic cleft palate), or families with heterogeneous multi-systemic (neuro)developmental disorders and of prevalent Italian ancestry (18/27, 64\%) (Table 1). Genetic analyses performed prior to WES in 32 probands (68\%) were inconclusive and laboratory tests prescribed in specific instances after clinical examination were negative, as summarized in Supplemental Table 1.

Notwithstanding differences in library preparation and capture, all WES experiments achieved comparable coverage which was adequate for the identification of homozygous variants with, on average, a mean coverage of $82.5 \mathrm{X}( \pm 27.2 \mathrm{X})$ and \% of bases covered above $20 \mathrm{X}$ of $86.3 \%( \pm 5.3 \%)$ (Supplemental Table 2). Mean number of exonic and canonical splice-site variants ( \pm 2 bp from exon/intron boundary) was 22,920 $( \pm 1,271)$ per individual, of which 8,853 $( \pm 461)$ were homozygous. Autozygosity-driven prioritization restricted the list to a mean of $931( \pm 477)$ variants within large 
ROHs including 38 uSAVs in 23 families (mean $1.6 \pm 0.7$ ) as detailed in Supplemental Table 3, of which $19( \pm 12)$ per individual were candidate clinical.

Diagnostic yield of the autozygosity-driven WES analysis. In 22 out of 47 families where we identified a variant classified as pathogenic (Table 2) or likely pathogenic (Table 3) according to our criteria, we claimed that we established diagnosis (47\%).

Nine variants were LOF while 11 were missense. Clinical significance of 4 missense variants was convincingly supported by ClinVar (Table 2), while one, ACP5 p.Q120R, was described in another Italian individual (Patient 21 in Briggs et al., 2016). MutpredSplice predicted 11 uSAVs within genes associated with autosomal recessive Mendelian diseases, 5 of which consistent with the patient's clinical presentation. Four were nonsynonymous changes already classified as pathogenic or likely pathogenic, three in established deafness genes (CDH23, COL9A2, PCDH15) and one in TRAPPC2L (Milev et al., 2018), while one was the novel synonymous NARS2 p.N90N variant. This unconventional splice-site variant was predicted to produce an Exonic Splicing Enhancer (ESE) loss and Exonic Splicing Silencer (ESS) gain and it was therefore added to the list of pathogenic variants. We subsequently confirmed that the functional consequence of this variant was NARS2 exon 9 skipping resulting in an out-of-frame truncated protein (p.N90HfsX4) by analysis of cDNA from patient's fibroblasts. With specific primers, we obtained a single NARS2 amplicon (318 bp) in all tested control tissues. In the patient, bands with different size indicated the activation of alternative splice sites, including those eliminating exon 9 (280 bp). Interestingly, patient's fibroblasts maintained the wild-type band, while skeletal muscle cells, which are one of the disease targets, lost it completely (Supplemental Figure $1 \mathrm{~A}, \mathrm{~B}$ ). To assess the presence of the NARS2 wt transcript, a Real-Time PCR was carried out on RNA from both fibroblasts and muscle biopsy: in the proband the wt transcript resulted expressed at very low levels with respect to controls in both tissues (Supplemental Figure $1 \mathrm{C}$ ). 
In two instances, the candidate clinical variants were found in association with phenotypes only partially overlapping the classical presentation ascribable to the genes. $D D C$ p.Arg375Cys was described to expand the phenotypic spectrum of aromatic aminoacid decarboxylase deficiency related to $D D C$ mutations (Graziano et al., 2015). MC2R p.L283R only partially explains the composite clinical picture in patient from family GME_14, since while it was assessed as causative of the glucocorticoid deficiency phenotype, it could not clarify patient's craniostenosis, which is a feature that has never been observed so far in $M C 2 R$-related disorders. In two further families, homozygous variants in two genes normally underlying autosomal dominant diseases, $R A D 21$ (p.A622T) and NOTCH3 (p.C966X), were demonstrated to cause recessive phenotypes that partially overlapped the dominant ones (Bonora et al., 2015; Pippucci et al., 2015).

We then searched for variants out of long ROHs, to uncover disease-related variants within shortmedium ROH. MYO15A p.Y393CfsX41, identified in three siblings affected with congenital deafness from family GME_17, was within a $\mathrm{ROH}$ ranging about $900 \mathrm{~Kb}$ and we previously demonstrated that it is a founder mutation in Oman (Palombo et al., 2017), the country from which this family originates. We also evaluated potentially pathogenic variants identified in mutation-negative families after previous steps, resulting in 20 candidate genes. For 3 of these genes (TRAPPC2L, SMPD4 and CCDC32) we were connected through Gene Matcher to colleagues that had identified defects in one of the same genes in patients presenting with convincingly overlapping phenotypes. We were thus able to collect a series of genetic and functional evidence to support novel disease-gene association which are described in separate publications (Milev et al., 2018; Magini et al., 2019; Harel et al., 2020). Thanks to these collaborative efforts we were eventually able to ascertain the causative role of these three variants and to upgrade their pathogenicity classification from potentially pathogenic to pathogenic (Table 2). Overall, with the MYO15A variants and variants that were initially classified as potentially pathogenic but were later assessed to be causative, we established diagnosis in 26/47 families (11/18 Italian families, 60\%; 15/29 GME families, 51\%) and we were thus able to increase 
the diagnostic yield to 55\% (26/47). Diagnostic yields varied substantially between the principal different disease groups, as we achieved genetic diagnosis in 8/12 of the probands with nonsyndromic congenital deafness (66\%), in 3/8 of those with syndromic cleft palate (37\%), and in 10/16 of those with neurodevelopmental disorders (62.5\%).

Among the remaining 17 novel candidate genes (Table 4), we deem that at least NDUFAF7 deserves a comment although we were not able to provide likewise evidence that it is causative of the patient's clinical manifestation. NDUFAF7 p.A278T was identified in a patient with a severe form of leukodystrophy with suspected mitochondrial origin. This gene, which has been functionally characterized only recently (Rhein et al., 2013), encodes a S-adenosylmethionine-dependent methyltransferase located in the mitochondrial matrix that symmetrically dimethylates the residue Arg-85 in the NDUFS2 subunit during the early stage of complex I assembly. Moreover, NDUFAF7 seems to be essential for normal vertebrate development as its knockout in mice is embryonically lethal (Zurita Rendón et al. 2014).

Proportion of autozygous genome and variants and evaluation of their impact on diagnostic yield. The cumulative length of individual long ROHs, assumed to be the autozygous genome, ranged from 62.6 $\mathrm{Mb}$ to $432 \mathrm{Mb}$ (mean: $253 \mathrm{Mb} \pm 97 \mathrm{Mb}$ ). Such a wide span in the autozygous genome is consistent with literature data reporting a $86 \mathrm{Mb}-345 \mathrm{Mb}$ range in the offspring to first cousin unions (Leutenegger et al., 2003). Although GME families had on average a larger autozygous genome than Italian families (266 Mb $\pm 98 \mathrm{Mb}$ vs. $231 \mathrm{Mb} \pm 96 \mathrm{Mb}$ ), there was substantial overlap in range (62.6 Mb-432 Mb vs. 96 Mb-406 Mb) resulting in no significant difference (p-value = 0.3096).

The autozygous genome positively correlated with the total number of variants in long $\mathrm{ROH}$ (Adjusted R-squared: 0.8103, p-value: <2.2e-16), as well as, although showing progressively weaker correlation, with the number of rare variants in long ROHs (Adjusted R-squared: 0.5711, p-value: 5.713e-11) or of only candidate clinical variants in long ROHs (Adjusted R-squared: 0.2364, p-value: 0.000153) (Supplemental Figure 2). 
GME individuals had a higher mean number of variants in long ROHs compared to individuals of Italian ancestry for all total, rare and candidate clinical variants, although this difference was not significant (total: 32390 vs. 16029 , mean 981.5 std \pm 483.6 vs. $843.6 \pm 465.1$, p-value $=0.3166$; rare: 1156 vs. $473,35 \pm 22.1$ vs. $24.9 \pm 14.3$, p-value =0.05105; candidate clinical: 126 vs. $68,3.8 \pm 2.7$ vs. $3.5 \pm 2.9$, p-value $=0.7766)$.

We eventually examined variables that could mark a difference between mutation-positive and mutation-negative families. First, since the mean depth of sequence coverage on target is commonly defined as a parameter of good experimental quality, we compared mean on-target coverage in mutation-positive (83.8X) and mutation-negative (80.5X) families finding no significant difference $(\mathrm{p}$-value $=0.6719)$. Differences in sequence coverage are not thus likely to determine failure or success to detect the diagnostic variant in this study.

We then wondered whether the proportion of autozygous genome and/or the proportion of candidate clinical variants in long ROHs was on average higher in families where a causative variant was identified. Mutation-positive families showed trend towards a higher proportion of autozygous genome compared to mutation-negative families (Figure 1) (282 Mb vs. 234 Mb; p-value = 0.08548) and a statistically significant higher number of candidate clinical variants (Figure 2) (5 vs. 2.5; pvalue $=0.003356$ ), this trend surviving even when diagnostic variants in mutation-positive families were excluded from the analysis (4 vs 2.5; p-value $=0.0698$ ). 


\section{Discussion}

In this study, a collection of 47 consanguineous families with occurrence of a variety of suspected genetic conditions underwent WES analysis under suspicion of an autozygosity-related autosomal recessive disease. Therefore, an autozygosity-driven bioinformatics workflow similar to previous studies (Makrythanasis et al., 2015; Harripaul et al., 2018), with the combined analysis of uSAVs by MutPredSplice, achieved an initial diagnostic yield of 47\% (22/47 families). This number includes a family displaying a non-canonical phenotype ascribable to DDC mutations (Graziano et al., 2015), as well as a proband who received a partial diagnosis. This proband had glucocorticoid deficiency explained by a $M C 2 R$ variant and sagittal synostosis, the most common form of craniostenosis known for a low contribution from monogenic forms (Wilkie et al., 2017), suggesting that the two defects are independent and that craniostenosis in this patient is not likely caused by a single variant. Moreover, in two families (Bonora et al., 2015; Pippucci et al., 2015) recessive variants were found in genes that are normally described in dominant clinically-overlapping phenotypes, a phenomenon observed also in other reports (Harel et al., 2016; Monies et al., 2017). In addition, identification of an ACP5 variant allowed clinical re-assessment of a patient initially diagnosed with pure hereditary spastic paraplegia with associated autoimmune haemolitic anemia. Involvement of ACP5, a gene associated with spondyloenchondrodysplasia with immune dysregulation (MIM 607944) which has spasticity among its clinical signs, motivated to perform X-rays in this patient. Radiologic findings revealed the presence of vertebra's platispondilia and epiphysial radiolucencies of the radius fully consistent with the ACP5-related phenotypic spectrum.

Interestingly, a homozygous uSAV in NARS2, otherwise discarded by canonical variant filtering workflows as it caused a synonymous codon change, was eventually demonstrated to be an actual LOF variant that by impacting the normal splicing of NARS2 exon 9 led to generation of an out-offrame transcript, and as such pathogenic (Supplemental Figure 1). As their functional effect is more difficult to predict than that of missense changes, synonymous variants and uSAVs in general are 
usually overlooked in WES studies. The autozygosity-driven strategy allowed to narrow the search space to long ROHs, thus shortening the list of MutPredSplice variants among which it was then easy to point to the NARS2 one. Since uSAVs can behave as LOF variants, it is becoming more and more urgent to investigate their role in autosomal recessive diseases and an autozygosity-driven analysis can be of great help in this task.

We were subsequently able to increase the diagnostic yield to 55\% in two ways. First, we looked for homozygous variants outside long ROHs. This revealed a MYO15A homozygous frame-shift small duplication that could explain the deafness phenotype, surrounded by a $849 \mathrm{~Kb}$ ROH shared by three Omani siblings. We demonstrated (Palombo et al., 2017) that this short-medium ROH reflected a founder haplotype introduced in Oman within the past 2-3 centuries and with an estimated carrier frequency of about $1 \%$ in Northern Oman, suggesting that focusing on large ROHs (Prasad et al., 2018; Wakeling et al., 2019) may cause to miss clinical variants that are not linked to long haplotypes arising as a result of recent parental relatedness. Second, gene matchmaking (Sobreira et al., 2015) allowed to establish candidate genes as disease-associated before they were published (Milev et al., 2018; Magini et al., 2019, Harel et al., 2020), with important implications for patient management and family counseling.

We achieved the highest diagnostic yield in probands with non-syndromic congenital deafness (66\%), exceeding that obtained by WES and microarray approaches combined (56\%) in another recent study on Australian non-consanguineous families (Downie et al., 2019), likely explained by the consanguinity of our families. Two studies that embraced consanguineous cohorts with multiple congenital anomalies and neurocognitive/neurodevelopmental disorders reported diagnostic yields of 38.8\% (Al-Dewick et al., 2019) and 46-54\% (Al-Dewick et al., 2019; Charng et al., 2016) for the two classes, respectively. These findings were comparable to our similar disease groups of syndromic CP (37\%) and neurodevelopmental disorders (62.5\%). 
A 55\% overall diagnostic yield is a striking result, but it is not unexpected in a WES study of consanguineous families. Makrythanasis et al., 2014 established diagnosis in 18/50 families of predominantly Arab ancestry (36\%) (Makrythanasis et al., 2014). Yavarna et al., 2105 detected pathogenic or likely pathogenic variants in 89/149 Qatari probands (60\%) (Yavarna et al., 2015). Charng et al., 2016 reported a potential molecular diagnosis in 17/31 families in Saudi Arabia (54.8\%) (Charng et al., 2016). Finally, WES was used as first-line diagnostic tool in Palestinian and Israeli Arab consanguineous families, reaching a diagnostic rate of 51\% (42/83) (Hengel et al., 2020). Similar high diagnostic rates were recently reported in large cohorts of consanguineous families: in a multicenter clinical exome study, Alfares et al., 2017 identified likely disease-causing variants in 222/454 probands (49\%) (Alfares et al., 2017), while Al-Dewik et al., 2019 reported the Clinical Exome Sequencing experience in Qatar with 246/509 probands receiving a molecular diagnosis (48.3\%) (Al-Dewik et al., 2019). In these studies, almost the entire cohorts have GME ancestry, which raises the question whether in our study GME patients, coming from countries with high inbreeding rates, received more autozygosity-related diagnoses than Italian patients. Indeed, the two groups contributed comparably to the achieved diagnostic yield. On average, the autozygous genome was larger (and the number of variants in long ROHs was higher) in GME than in Italian patients, maybe a consequence of the closer parental relatedness of GME parents. However, these differences were not significant, indicating that patients belonging to a GME population known to have high inbreeding levels and/or consanguinity rates does not necessarily have significant autozygosity excess compared to patients from consanguineous families of European ancestry. Moreover, going down from total to rare and then to candidate clinical variants, the excess seen in GME patients is progressively smaller, suggesting that the augmented autozygosity does not necessarily reflects in significant over-burden of deleterious variants as also indicated by the progressively weaker correlation between extent of the autozygous genome and the total/rare/candidate clinical variants in the whole sample. 
We thus wanted to understand what could be significantly different between mutation-positive and mutation-negative patients, to get an insight into reasons that caused failure to identify the causative variant. We advanced three hypotheses:

- $\quad$ differences in exome target coverage could explain why we failed to establish diagnosis in the remaining 21 families;

- $\quad$ although most family loops had 1st cousins parents, extent of the autozygous genome varied substantially from family to family: families with smaller autozygous genome are therefore less likely to carry an autozygosity-related variant than families with larger autozygous genome;

- $\quad$ even with comparable extent of the autozygous genome, in some families there may be higher occurrence of deleterious variants in shared parental haplotypes resulting in a higher chance to inherit an autozygosity-related causative variant.

While there was no apparent discrepancy in exome target coverage that could justify detection failures, we noticed that mutation-positive probands showed a trend towards augmented autozygosity and, most importantly, had significantly more autozygous deleterious variants. Notably, the extent of the "autozygome" was recently observed to correlate with the detection rate of recessive diseasecausing variants in patients from a high-consanguinity community (Hengel et al., 2020). Moreover, probands of Pakistani ancestry in the Deciphering Developmental Disorders (DDD) study who were characterized by higher levels of genomic inbreeding also showed an increased rate in the diagnosis of autosomal recessive diseases (Martin et al., 2018). These observations have important implications, as they suggest that probands with lower degrees of genomic autozygosity, irrespective of the high pedigree-based inbreeding coefficient, are less likely to have their disease explained through an autozygosity-driven approach. The observation is consistent with the recent finding in 1000 genomes project data that as the genome is progressively covered by autozygous regions the rate in gain of homozygous damaging alleles outpaces that for less damaging alleles (Pemberton and Szpiech, 2018). 
This is well illustrated by two of our families where all patients had an extent of the autozygous genome below the median value of our cohort (265 Mb). First, the three siblings from family GME_17 with the MYO15A variant, a founder mutation that is not linked to recent common parental haplotypes, had relatively low autozygosity levels (ranging 62-204 Mb) although these individuals belong to a population with one of the highest inbreeding level worldwide (Islam, 2012).

Second, in one of the Iranian probands the review of clinical images suggested the hypothesis of Kabuki syndrome: ad-hoc inspection of KMT2D revealed a pathogenic LOF mutation that turned out to be de novo after parental testing. Notably, also this patient had low autozygosity levels (214 Mb) and was the only one with a pathogenic/likely pathogenic variant in a known dominant gene. Assessment of this case was facilitated by a straightforward diagnosis after review, but it suggests that it is worth to consider that probands with low autozygosity levels may rather be affected by non autozygosity-related diseases. That probands from consanguineous families may have e.g. a dominant disorder is an obvious consideration; however the hypothesis that it is more likely to occur while autozygosity levels decrease may have consequences on study design (trio to have higher chance of detecting de novo variants in place of proband-only sequencing that can instead be adequate when searching for homozygous causative variants).

In conclusion, we highlighted the utility of autozygosity-driven WES analysis in an European population, where consanguinity is not widespread cultural tradition. Analysis of uSAVs, inspection of short-medium ROHs and genetic match-making were key in our study to improve diagnostic yield. Irrespective of patient's population or ethnicity, augmented genome autozygosity reflected in higher diagnostic chance under the assumption of an autozygosity-related disease. These observations may influence study design and clinical prioritization of genetic variants in autozygosity-driven WES studies. 


\section{Conflict of Interest}

The authors declare that they have no conflict of interest.

\section{Acknowledgment}

NH and MS are supported by the Deputy of Research, Isfahan University of Medical Sciences, Isfahan, Iran (grant no. 189087). We are thankful to Dr.ssa Alessandra Maresca, IRCCS Istituto delle Scienze Neurologiche di Bologna, UOC Clinica Neurologica, Bologna, Italy, for her assistance with the Real-Time data analysis.

\section{Author Contributions}

FP performed research, analyzed and interpreted data. FP, TP and CG wrote the manuscript. CG, NAW, NN, GS, CLM, GC, DMC, SG, MNAK, MAK, MS, EG, AB and VC collected patients' clinical data and contributed the DNA samples. CM and PM performed the experiments. TP, GR and MS designed and conceived the general overview of the study. All the authors critically revised the manuscript and accepted the final version. 


\section{References}

Alazami AM, Patel N, Shamseldin HE et al. (2015) Accelerating novel candidate gene discovery in neurogenetic disorders via whole-exome sequencing of prescreened multiplex consanguineous families. Cell Rep, 10(2), 148-161. doi: 10.1016/j.celrep.2014.12.015

Aldahmesh MA, Mohamed JY, Alkuraya HS, Verma IC, Puri RD, Alaiya AA, Rizzo WB, Alkuraya FS. (2011) Recessive mutations in ELOVL4 cause ichthyosis, intellectual disability, and spastic quadriplegia. Am J Hum Genet, 89(6), 745-750. doi: 10.1016/j.ajhg.2011.10.011

Al-Dewik N, Mohd H, Al-Mureikhi M et al. (2019) Clinical exome sequencing in 509 Middle Eastern families with suspected Mendelian diseases: The Qatari experience. Am J Med Genet Part A, 179A, 927-935. doi: 10.1002/ajmg.a.61126

Alfares A, Alfadhel M, Wani T et al. (2017) A multicenter clinical exome study in unselected cohorts from a consanguineous population of Saudi Arabia demonstrated a high diagnostic yield. Mol Genet Metab, 121(2), 91-95. doi: 10.1016/j.ymgme.2017.04.002

Becker J, Semler O, Gilissen C et al. (2011) Exome sequencing identifies truncating mutations in human SERPINF1 in autosomal-recessive osteogenesis imperfecta. Am J Hum Genet, 88(3), 362371. doi: 10.1016/j.ajhg.2011.01.015

Bittles AH. (2001) Consanguinity and its relevance to clinical genetics. Clin Genet, 60, 89-98. doi: 10.1034/j.1399-0004.2001.600201.x 
Bonora E, Bianco F, Cordeddu L et al. (2015) Mutations in RAD21 Disrupt Regulation of APOB in Patients with Chronic Intestinal Pseudo-obstruction. Gastroenterology, 148(4), 771-782.e11. doi: 10.1053/j.gastro.2014.12.034

Briggs TA, Rice GI, Adib N et al. (2016) Spondyloenchondrodysplasia Due to Mutations in ACP5: A Comprehensive Survey. J Clin Immunol, 36(3), 220-34. doi: 10.1007/s10875-016-0252-y

Cavalli-Sforza LL, Moroni A and Zei G. (2004) Consanguinity, inbreeding, and genetic drift in Italy. Inbreeding in Italy. Monographs in Population Biology. Levin S. A. and Horn H. S. (eds). Princeton University Press, Oxford, UK, 320pp

Charng WL, Karaca E, Coban Akdemir Z et al. (2016) Exome sequencing in mostly consanguineous Arab families with neurologic disease provides a high potential molecular diagnosis rate. BMC Med Genomics, 9(1), 42. doi: 10.1186/s12920-016-0208-3

Chiang PW, Wang J, Chen Y et al. (2012) Exome sequencing identifies NMNAT1 mutations as a cause of Leber congenital amaurosis. Nat Genet, 44(9), 972-974. doi: 10.1038/ng.2370

Chong JX, Buckingham KJ, Jhangiani SN et al. (2015) The Genetic Basis of Mendelian Phenotypes: Discoveries, Challenges, and Opportunities. Am J Hum Genet, 97(2), 199-215. doi: 10.1016/j.ajhg.2015.06.009 
DePristo MA, Banks E, Poplin R et al. (2011) A framework for variation discovery and genotyping using next-generation DNA sequencing data. Nat Genet, 43(5), 491-501. doi: 10.1038/ng.806

Downie L, Halliday J, Burt R et al. (2020) Exome sequencing in infants with congenital hearing impairment: a population-based cohort study. Eur J Hum Genet, 28(5), 587-596. doi: 10.1038/s41431-019-0553-8

Graziano C, Wischmeijer A, Pippucci T et al. (2015) Syndromic intellectual disability: a new phenotype caused by an aromatic amino acid decarboxylase gene (DDC) variant. Gene, 559(2), 1448. doi: 10.1016/j.gene.2015.01.026

Harel T, Yoon WH, Garone C et al. (2016) Recurrent De Novo and Biallelic Variation of ATAD3A, Encoding a Mitochondrial Membrane Protein, Results in Distinct Neurological Syndromes. Am $J$ Hum Genet, 99(4), 831-845. doi: 10.1016/j.ajhg.2016.08.007

Harel T, Griffin JN, Arbogast T et al. (2020) Loss of function mutations in CCDC32 cause a congenital syndrome characterized by craniofacial, cardiac and neurodevelopmental anomalies. Hum Mol Genet, 2020 Apr 20. pii: ddaa073. doi: 10.1093/hmg/ddaa073. [Epub ahead of print]

Harripaul R, Vasli N, Mikhailov A et al. (2018) Mapping autosomal recessive intellectual disability: combined microarray and exome sequencing identifies 26 novel candidate genes in 192 consanguineous families. Mol Psychiatry, 23(4), 973-984. doi: 10.1038/mp.2017.60 
Hengel H, Buchert R, Sturm M et al. (2020) First-line exome sequencing in Palestinian and Israeli Arabs with neurological disorders is efficient and facilitates disease gene discovery. Eur J Hum Genet 2020 Mar 25. doi: 10.1038/s41431-020-0609-9. [Epub ahead of print]

Islam MM. (2012) The practice of consanguineous marriage in Oman: prevalence, trends and determinants. J Biosoc Sci, 44, 571-594. doi: 10.1017/S0021932012000016

Lander ES, Botstein D. (1987) Homozygosity mapping: a way to map human recessive traits with the DNA of inbred children. Science, 236, 1567-1570. doi: 10.1126/science.2884728

Leutenegger AL, Prum B, Genin E, Verny C, Lemainque A, Clerget-Darpoux F, Thompson EA. (2003) Estimation of the inbreeding coefficient through use of genomic data. Am J Hum Genet, 73(3), 516-523. doi: 10.1086/378207

Li H, Durbin R. (2009) Fast and accurate short read alignment with Burrows-Wheeler transform. Bioinformatics, 25, 1754-1760. doi: 10.1093/bioinformatics/btp324

Magi A, Tattini L, Palombo F et al. (2014) H3M2: Detection of run oh Homozygosity from wholeexome sequencing data. Bioinformatics, 30(20), 2852-9. doi: 10.1093/bioinformatics/btu401 
Magini P, Pippucci T, Tsai IC et al. (2014) A mutation in PAK3 with a dual molecular effect deregulates the RAS/MAPK pathway and drives an X-linked syndromic phenotype. Hum Mol Genet, 23(13), 3607-17. doi: 10.1093/hmg/ddu070

Magini P, Smits DJ, Vandervore L et al (2019) Loss of SMPD4 Causes a Developmental Disorder Characterized by Microcephaly and Congenital Arthrogryposis. Am J Hum Genet, pii: S00029297(19)30309-X. doi: 10.1016/j.ajhg.2019.08.006

Makrythanasis P, Nelis M, Santoni FA et al. (2014) Diagnostic Exome Sequencing to Elucidate the Genetic Basis of Likely Recessive Disorders in Consanguineous Families. Hum Mutat, 35(10), 12031210. doi: 10.1002/humu.22617

Martin HC, Jones WD, McIntyre R et al. (2018) Quantifying the contribution of recessive coding variation to developmental disorders. Science, 362(6419), 1161-1164.

McQuillan R, Leutenegger AL, Abdel-Rahman R et al. (2008) Runs of homozygosity in European populations. Am J Hum Genet, 83(3), 359-372.

Milev MP, Graziano C, Karall D et al. (2018) Bi-allelic mutations in TRAPPC2L result in a neurodevelopmental disorder and have an impact on RAB11 in fibroblasts. J Med Genet, 55(11), 753764. doi: 10.1136/jmedgenet-2018-105441 
Monies D, Abouelhoda M, AlSayed M et al. (2017) The landscape of genetic diseases in Saudi Arabia based on the first 1000 diagnostic panels and exomes. Hum Genet, 136(8), 921-939. doi: 10.1007/s00439-017-1821-8

Monies D, Maddirevula S, Kurdi W et al. (2017) Autozygosity reveals recessive mutations and novel mechanisms in dominant genes: implications in variant interpretation. Genet Med, 19(10), 11441150. doi: 10.1038/gim.2017.22

Ng SB, Buckingham KJ, Lee C et al. (2010) Exome sequencing identifies the cause of a Mendelian disorder. Nat Genet, 42(1), 30-35. doi: 10.1038/ng.499

Palombo F, Al-Wardy N, Ruscone GA et al. (2017) A novel founder MYO15A frameshift duplication is the major cause of genetic hearing loss in Oman. J Hum Genet, 62(2), 259-264. doi: 10.1038/jhg.2016.120.

Pemberton TJ, Absher D, Feldman MW, Myers RM, Rosenberg NA, Li JZ. (2012) Genomic Patterns of Homozygosity in Worldwide Human Populations. Am J Hum Genet, 91(2), 275-292. doi: 10.1016/j.ajhg.2012.06.014

Pemberton TJ, Szpiech ZA. (2018) Relationship between Deleterious Variation, Genomic Autozygosity, and Disease Risk: Insights from the 1000 Genomes Project. Am J Hum Genet, 102(4), 658-675. doi: 10.1016/j.ajhg.2018.02.013 
Pippucci T, Parmeggiani A, Palombo F et al. (2013) A novel null homozygous mutation confirms CACNA2D2 as a gene mutated in epileptic encephalopathy. PLoS One, 8(12), e82154. doi: 10.1371/journal.pone.0082154

Pippucci T, Maresca A, Magini P et al. (2015) Homozygous NOTCH3 null mutation and impaired NOTCH3 signaling in recessive early-onset arteriopathy and cavitating leukoencephalopathy. EMBO Mol Med, 7(6), 848-58. doi: 10.15252/emmm.201404399

Prasad A, Sdano MA, Vanzo RJ et al. (2018) Clinical utility of exome sequencing in individuals with large homozygous regions detected by chromosomal microarray analysis. BMC Med Genet, 19(1), 46. doi: 10.1186/s12881-018-0555-3

Ramos EM, Roca A, Chumchim N et al. (2019) Primary familial brain calcification caused by a novel homozygous MYORG mutation in a consanguineous Italian family. Neurogenetics, 20(2), 99-102. doi: 10.1007/s10048-019-00571-8

Rentzsch P, Witten D, Cooper GM, Shendure J, Kircher M. (2018) CADD: predicting the deleteriousness of variants throughout the human genome. Nucleic Acids Res, 47(D1), D886-D894 doi: 10.1093/nar/gky1016.

Rhein VF, Carroll J, Ding S, Fearnley IM, Walker JE. (2013) NDUFAF7 methylates arginine 85 in the NDUFS2 subunit of human complex I. J Biol Chem, 288(46), 33016-26. doi: 10.1074/jbc.M113.518803 
Richards S, Aziz N, Bale S et al. (2015) Standards and guidelines for the interpretation of sequence variants: a joint consensus recommendation of the American College of Medical Genetics and Genomics and the Association for Molecular Pathology. Genet Med, 17(5), 405-24. doi: 10.1038/gim.2015.30

Shaheen R, Faqeih E, Sunker A et al. (2011) Recessive mutations in DOCK6, encoding the guanidine nucleotide exchange factor DOCK6, lead to abnormal actin cytoskeleton organization and AdamsOliver syndrome. Am J Hum Genet, 89(2), 328-333. doi: 10.1016/j.ajhg.2011.07.009

Smith CAB. (1974) Measures of homozygosity and inbreeding in populations. Ann Hum Genet, 37(4), 377-391. doi: 10.1111/j.1469-1809.1974.tb01844.x

Sobreira NL, Cirulli ET, Avramopoulos D et al. (2010) Whole-genome sequencing of a single proband together with linkage analysis identifies a Mendelian disease gene. PLoS Genet, 6(6), e1000991. doi: 10.1371/journal.pgen.1000991

Sobreira N, Schiettecatte F, Valle D, Hamosh A. (2015) GeneMatcher: A Matching Tool for Connecting Investigators with an Interest in the Same Gene. Hum Mutat, 36(10), 928-30. doi: 10.1002/humu.22844. 
Spataro R, Kousi M, Farhan SMK et al. (2019) Mutations in ATP13A2 (PARK9) are associated with an amyotrophic lateral sclerosis-like phenotype, implicating this locus in further phenotypic expansion. Hum Genomics, 13(1), 19. doi: 10.1186/s40246-019-0203-9

Wakeling MN, Laver TW, Wright CF et al. (2019) Homozygosity mapping provides supporting evidence of pathogenicity in recessive Mendelian disease. Genet Med, 21(4), 982-986. doi: 10.1038/s41436-018-0281-4

Wang K, Li M, Hakonarson H. (2010) ANNOVAR: Functional annotation of genetic variants from next-generation sequencing data. Nucleic Acids Research, 38(16), e164. doi: 10.1093/nar/gkq603

Wilkie AOM, Johnson D, Wall SA. (2017) Clinical Genetics of Craniosynostosis. Curr Opin Pediatr, 29(6), 622-628. doi: 10.1097/MOP.0000000000000542

Yavarna T, Al-Dewik N, Al-Mureikhi M et al. (2015) High diagnostic yield of clinical exome sequencing in Middle Eastern patients with Mendelian disorders. Hum Genet, 134(9), 967-80. doi: 10.1007/s00439-015-1575-0

Zurita Rendón O, Silva Neiva L, Sasarman F, Shoubridge EA. (2014) The arginine methyltransferase NDUFAF7 is essential for complex I assembly and early vertebrate embryogenesis. Hum Mol Genet, 23(19), 5159-70. doi: 10.1093/hmg/ddu239. 
Figure 1. Boxplot showing proportion of autozygous genome in mutation -positive and -negative families. Mutation-positive families showed trend towards a higher proportion of autozygous genome compared to mutation-negative families (282 Mb vs. $234 \mathrm{Mb}$; p-value $=0.08548$ ).

Figure 2. Boxplot showing proportion of autozygous clinical candidate variants in mutation positive and -negative families. Mutation-positive families showed a statistically significant higher number of autozygous pathogenic/likely pathogenic/potentially pathogenic variants (5 vs. 2.5; pvalue $=0.003356)$.

Supplemental figure 1. Analysis and expression of NARS2 transcripts from control's and patient's tissues. A. Gel electrophoresis of NARS2 cDNA amplicons from brain (B) and colon (C) transcript libraries, and from skeletal muscle cells and fibroblasts of a healthy individual (M and F) and patient (Pt M and Pt F). Bands corresponding to wild-type (wt, 318 bp) and exon 9 skipped (altered, $280 \mathrm{bp)}$ transcripts are indicated by red arrows. B. Sanger sequencing of NARS2 cDNA amplicons. In this panel, NARS2 cDNA reference sequence and electropherograms of wild-type and incorrectly spliced transcripts are reported. Exon 8 - exon 10 junction and exon 9 skipping are indicated by red lines. C. mRNA content of the proband was evaluated by Real Time-PCR; ACTB was used for normalization. NARS2 mRNA resulted strongly reduced in the proband both in fibroblasts and in muscle tissue respect to the controls. Four fibroblasts and three muscle biopsies were used as control; data are shown as mean \pm standard error.

Supplemental figure 2. Correlation between autozygous genome and autozygous variants. A fitted linear regression model was used to identify the positive correlation between autozygous genome and autozygous variants. A. Total number of autozygous variants; Adjusted R-squared: 
0.8103, p-value: < 2.2e-16. B. Rare autozygous variants; Adjusted R-squared: 0.5711, p-value: 5.713e-11. C. Candidate clinical autozygous variants; Adjusted R-squared: 0.2364, p-value: 0.000153 . 
Figure 1.

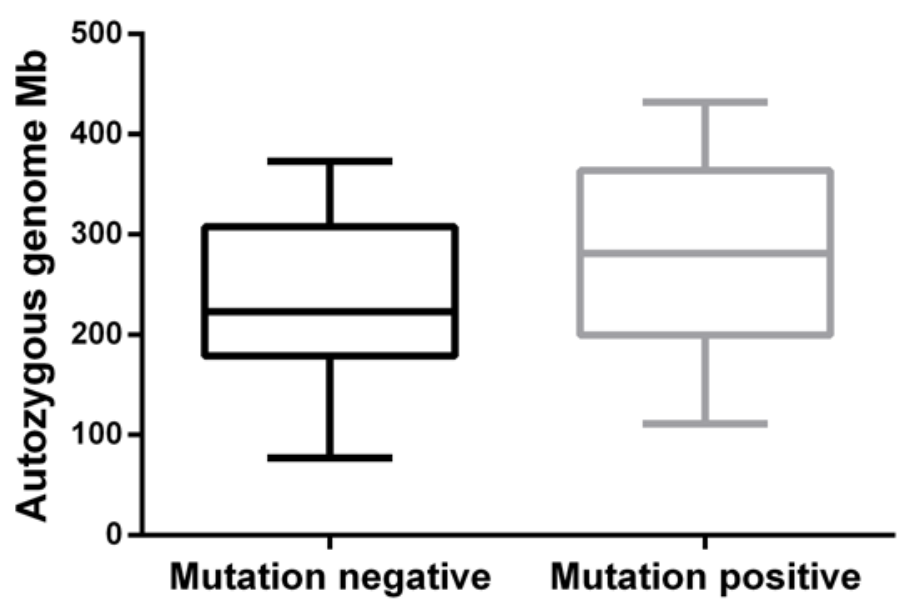

Figure 2

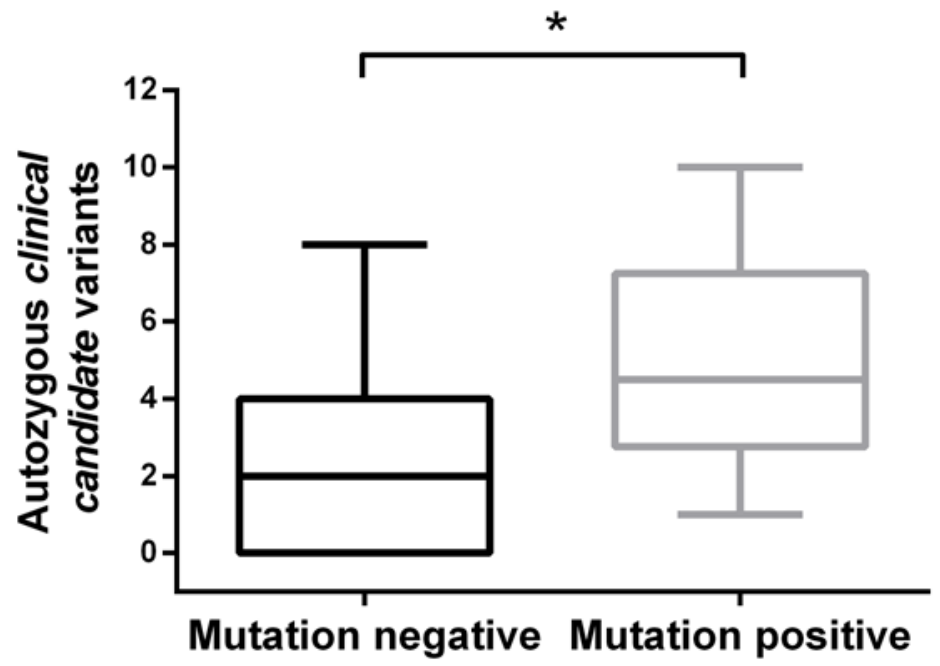




\begin{tabular}{|c|c|c|c|c|}
\hline Family & Country of origin & $\begin{array}{l}\text { Closest relationship between } \\
\text { parents of probands }\end{array}$ & Phenotype & WES (affected) \\
\hline ITA_1 & | Italy & First cousins & $\begin{array}{l}\text { Short stature, facial hirsutism, alopecia, } \\
\text { dyslipidemia and extreme insulin resistance }\end{array}$ & $1^{\dagger}(1)$ \\
\hline ITA_2 & Italy & Second cousins once removed & ID & $1^{\dagger}(1)$ \\
\hline ITA_3 & Italy & First cousins & ID & $1^{\dagger}(1)$ \\
\hline ITA_4 & Italy & First cousins & Mitochondrial DNA depletion syndrome & $1^{*}(1)$ \\
\hline ITA_5 & Italy & First cousins & Epileptic Encephalopathy & $1^{*}(1)$ \\
\hline ITA_6 & Italy & Second cousins & $\begin{array}{l}\text { Metabolic encephalomyopathy mitochondrial } \\
\text { suspected }\end{array}$ & $1^{\ddagger}(1)$ \\
\hline ITA_7 & Italy & Second cousins & Syndromic ID & $2^{*}(2)$ \\
\hline ITA_8 & Italy & First cousins & Bilateral optic neuritis & $2^{*}(1)$ \\
\hline ITA_9 & Italy & First cousins & Reversible COX deficiency & $2^{*}(1)$ \\
\hline ITA_10 & Italy & First cousins & HSP and AIHA & $1^{\pi}(1)$ \\
\hline ITA_11 & Italy & First cousins & Leukoencephalopathy & $1^{\S}(1)$ \\
\hline ITA_12 & Italy & First cousins & Gamma-globulinemia & $1^{\S}(1)$ \\
\hline ITA_13 & Italy & First cousins & Corpus callosum agenesia & $1^{\dagger}(1)$ \\
\hline
\end{tabular}




\begin{tabular}{|c|c|c|c|c|}
\hline ITA_14 & Italy & First cousins once removed & Microphtalmia and hypoacusia & $1^{\ddagger}(1)$ \\
\hline ITA_15 & Italy & First cousins & HSP, diplopia and nistagmo & $11^{\pi}(1)$ \\
\hline ITA_16 & Italy & Second cousins & ID (CNS malformation) & $1^{\dagger}(1)$ \\
\hline ITA_17 & Italy & First cousins & Diaphragmatic hernia & $1^{\ddagger}(1)$ \\
\hline ITA_18 & Italy & First cousins & Neuroaxonal dystrophy & $1^{\dagger}(1)$ \\
\hline GME_1 & Oman & First cousins & Deafness & $1^{\ddagger}(1)$ \\
\hline GME_2 & Oman & First cousins & Deafness & $1^{\ddagger}(1)$ \\
\hline GME_3 & Oman & First cousins & Deafness & $1^{\ddagger}(1)$ \\
\hline GME_4 & Oman & First cousins & Deafness & $1^{\ddagger}(1)$ \\
\hline GME_5 & Oman & First cousins & Deafness & $1^{\ddagger}(1)$ \\
\hline GME_6 & Oman & First cousins & Deafness & $1^{\ddagger}(1)$ \\
\hline GME_7 & Oman & First cousins & Deafness & $1^{\ddagger}(1)$ \\
\hline GME_8 & Oman & First cousins & Deafness & $1^{\ddagger}(1)$ \\
\hline GME_9 & Oman & First cousins & Deafness & $1^{\ddagger}(1)$ \\
\hline
\end{tabular}




\begin{tabular}{|c|c|c|c|c|}
\hline GME_10 & Oman & First cousins & Deafness & $1^{\ddagger}(1)$ \\
\hline GME_11 & Oman & First cousins & Deafness & $1^{\ddagger}(1)$ \\
\hline GME_12 & Turkey & First cousins & CIPO & $2^{\S}(2)$ \\
\hline GME_13 & Morocco & First cousins & HSP & $2^{\ddagger}(2)$ \\
\hline GME_14 & Pakistan & First cousins & Craniostenosis and glucocorticoid deficiency & $1^{\ddagger}(1)$ \\
\hline GME_15 & Bangladesh & First cousins & $\begin{array}{l}\text { Severe psychomotor delay, aggression, early } \\
\text { puberty }\end{array}$ & $1^{\ddagger}(1)$ \\
\hline GME_16 & Pakistan & First cousins & $\begin{array}{l}\text { Microcephaly, mild developmental delay, atrial } \\
\text { septal defect and multicystic left kidney }\end{array}$ & $1^{\ddagger}(1)$ \\
\hline GME_17 & Oman & First cousins & Deafness & $3^{\#}(3)$ \\
\hline GME_18 & Morocco & First cousins once removed & Lissencephaly & $2^{\ddagger}(1)$ \\
\hline GME_19 & Iran & First cousins & Syndromic CP & $1^{\ddagger}(1)$ \\
\hline GME_20 & Iran & First cousins once removed & Syndromic CP & $1^{\ddagger}(1)$ \\
\hline GME_21 & Iran & First cousins & Syndromic CP & $1^{\ddagger}(1)$ \\
\hline GME_22 & Iran & First cousins & Syndromic CP & $1^{\ddagger}(1)$ \\
\hline
\end{tabular}




\begin{tabular}{|c|c|c|c|c|}
\hline GME_23 & Iran & First cousins & Syndromic CP & $1^{\ddagger}(1)$ \\
\hline GME_24 & Iran & First cousins & Syndromic CP & $1^{\ddagger}(1)$ \\
\hline GME_25 & Iran & First cousins & Syndromic CP & $1^{\ddagger}(1)$ \\
\hline GME_26 & Iran & First cousins & Syndromic CP & $1^{\ddagger}(1)$ \\
\hline GME_27 & Pakistan & First cousins & Leukodystrophy & $1^{\pi}(1)$ \\
\hline GME_28 & Saudi Arabia & First cousins & ARMs & $1^{\ddagger}(1)$ \\
\hline GME_29 & Iran & First cousins & THC & $1^{\dagger}(1)$ \\
\hline
\end{tabular}




\section{Supplemental Material}

\section{Clinical description of selected families with confirmed or likely diagnosis}

\section{ITA_1 (POC1A)}

The proband was a 42-year old woman from Southern Italy, born from healthy first cousins. She has a primordial dwarfism, skeletal dysplasia, facial dysmorphisms, extreme dyslipidemia with insulin resistance and fatty liver.

During her childhood, she presented with acantosis nigricans in the neck region (7-8 years of age), premature menarche (9 years of age) and hands with dorsal swelling of the soft tissues. Until adolescence, foot also showed a dorsal bulge and she was diagnosed with diabetes (14 years of age). At the last evaluation (42 years of age), she has a worsening of acanthosis nigricans, short stature (138 cm, -4 SD), and normal intelligence. She also showed facial dysmorphism with micrognathia, prominent nose, low set ears, facial hirsutism, alopecia and thinning hair and eyebrows, in absence of nail and bone hypoplasia. At 40 years of age, she developed amenorrhea in the absence of a pituitary lesion. She presented lumbar lordosis, a dysplastic pelvis, mild knee arthrosis and bilateral patella osteosclerosis.

\section{ITA_4 (POLG)}

This 67-year old female, born from consanguineous parents, presented chronic progressive external ophthalmoplegia (CPEO) and dysphagia since 45 years of age, and generalized muscle weakness (more proximal) since she was 47 . Muscular symptoms progressively worsened over time. Since 56 years of age she also presented bilateral bradykinesia and postural tremor at upper limbs. Neurological examination showed myopathic face, CPEO, limb girdle hypotrophy, generalized hyposthenia at upper and lower limbs, hypopallesthesia, absent deep tendon reflexes, hypomimia and hypophonia, 
plastic rigidity of the upper limbs, bilateral bradykinesia, postural tremor of upper limbs, positive Romberg sign, bradykinetic and ataxic gait with reduced arm swing and pes cavus.

A therapy with l-DOPA (100 mg x 3/day) was started with improvement of tremor and bradykinesia (UPDRS=11; H\&Y: 1.5). She suddenly died at 67 ys and the cause of death was never ascertained.

Muscle biopsy showed increased variability of muscle fiber size, ragged red fibers (RRFs), some COX negative fibers and nuclear centralization. Proton magnetic resonance spectroscopy $\left({ }^{1} \mathrm{H}-\mathrm{MRS}\right)$ showed mild ventricular accumulation of lactic acid and she had diffuse mild cortical atrophy at brain MRI. Venous lactic acid after standardized exercise was slightly elevated $22.5 \mathrm{mg} / \mathrm{dl}$ (n.v. 5-22 $\mathrm{mg} / \mathrm{dl})$.

Audiometry showed sensorineural deafness. Optical coherence tomography of the optic nerve was normal. Cognitive tests showed multi-domain cognitive impairment. Electromyography showed moderate axonal sensorimotor (predominantly sensory) polyneuropathy. Polysomnography disclosed the presence of periodic limb movements. [123] MIBG Myocardial scintigraphy revealed defective sympathetic innervation and skin biopsy the presence of small fiber neuropathy with somatic and autonomic involvement.

\section{ITA_9 (NARS2)}

This 22-year-old male was born from consanguineous parents (1st degree cousins). Since birth he presented dysphagia. Psychomotor development was within normal limits in the first months of life, but at 7-8 months sensorineural deafness was noted, which was confirmed by audiometry performed at 14 months. At 11 months, after a febrile episode with fever over $40^{\circ} \mathrm{C}$ he presented psychomotor regression and hypotonia. Since 2 years of age he also developed upper limb tremor. Language and sphincteric control were never acquired. Neurological examination at 3 years of age showed hypoacusia, absence of language, hypotonia, hypotrophia, muscle weakness at lower limbs, brisk 
deep tendon reflexes, ataxic gait possible only with assistance with lower limb hyperextension. Brain MRI at that time was within normal limits. Electroencephalogram revealed photoparoxysmal response and left parietal spike and wave epileptiform discharges. Visual evoked potentials demonstrated delayed cortical responses. Fundus oculi was normal. Motor evoked potentials showed increased central conduction time at upper and lower limbs. Electroneurography was normal. Lactic acid was abnormally elevated, $27 \mathrm{mg} / \mathrm{dl}$ (nv 5-22 mg/dl). Muscle biopsy showed mitochondrial subsarcolemmal proliferation, diffuse presence of COX negative fibers and lipid droplets. Biochemical evaluation of muscle respiratory complexes revealed a generalized reduction of enzyme activities, including complex II. Mitochondrial DNA depletion (about 40\%) was also evident.

In the following years gait and dysphagia progressively worsened, and at 14 years of age the patient was wheelchair bound. At 17 years of age a second muscle biopsy was relevant for frequent increase of subsarcolemmal SDH staining, but only a few COX negative fibers, thus documenting a partial recovery of COX deficiency.

At 19 years of age neurological examination showed severe deafness, absence of spontaneous language, severe hypotrophy, spastic hypertonus, brisk deep tendon reflexes, myoclonus at upper limbs, multiple tendineous retractions with equinus foot bilaterally. $\mathrm{H}^{1}$ Brain MRI spectroscopy showed the presence of asymmetry of hippocampus with associated abnormal signal and mammillary bodies (left smaller than right) in the absence of pathological lactate in the brain ventricles.

\section{ITA_10 (ACP5)}

The proband was referred to our genetic unit when she was 10 . She was the second child of consanguineous (first cousins) healthy parents; family history was unremarkable. She was born from spontaneous delivery after an uneventful pregnancy; birth parameters were in the normal range. 
From the first months of life, tremors of the lower limbs and a global developmental delay were noted. Brain MRI at two years showed a myelinization delay of both cerebral and cerebellar cortex, with no parenchymal abnormalities. She subsequently developed spasticity of the lower limbs and short stature (-3,22 SD at 10 years, $-4,57$ SD at 14 years). At 10 years a mild neutropenia was noticed, followed by worsening of total white blood cell count, moderate neutropenia and lymphopenia; lymphocyte typing showed a moderate reduction of all T cell subtypes, and mild reduction of B cells. She developed autoimmune haemolytic anaemia at 11 years old.

Karyotype (on both amniotic fluid and blood sample) was 46, XX, no mutation in SPG7, SPG5, SPG11 and SPG15 were identified by Sanger sequencing.

After the diagnosis of SPENCD by exome sequencing (p.Q120R in the ACP5 gene), metaphyseal and spinal changes (marked platyspondyly with irregular plates) were confirmed on X-rays.

\section{ITA_16 (TRAPPC9)}

A nine-year-old girl at the time of our last examination was the second child of healthy Italian consanguineous parents. Family history is unremarkable and her older brother is healthy. She was born at term after an uneventful pregnancy. She showed a severe Intellectual Disability with absent speech, stereotypic movements and a self-injurious behavior. Motor milestones were markedly delayed and unassisted walking was possible at five years. She had normal growth with progressive microcephaly. She never experienced seizures. Brain imaging showed ponto-cerebellar hypoplasia, thinning of corpus callosum, hypomyelination. TRAPPC9 mutations are a very rare cause of recessive ID with brain abnormalities, perfectly consistent with the phenotype of this patient.

GME_14 (MC2R) 
A seven-month-old boy was referred to a genetic evaluation for the presence of a sagittal craniosynostosis. He was the fourth child of consanguineous parents from Pakistan. He was born at term after a pregnancy complicated by gestational diabetes and hypothyroidism. He had an older healthy sister, but two sisters had died, one for unknown reasons in the first day of life, another one at four months and was reported to suffer of infantile spasms.

The baby had scaphocephaly which required surgical correction, umbilical hernia, mild thinning of corpus callosum. At six months, he had an episode of febrile seizures with hypoglycemia which was not further investigated. At 30 months, his speech was limited to a few simple words, his growth was above the 97th percentile (height $102 \mathrm{~cm}$, weight $18.5 \mathrm{~kg}$, head circumference $53.5 \mathrm{~cm}$ ), and he was reported to have suffered several febrile seizures during a visit in Pakistan. While WES data were under analysis, he suffered again of febrile seizures, with hypoglycemia and increased ACTH levels. A primary adrenal insufficiency was suspected and WES analysis allowed to identify a MC2R homozygous mutation. Replacement therapy with oral hydrocortisone was immediately instituted.

\section{GME_16(CENPF)}

A two-year-old girl came to our attention for syndromic microcephaly. She was the only child of consanguineous healthy parents (first cousins); no family members with congenital malformations or developmental delay were reported. She was born at 34 weeks from C-section due to IUGR, fetal cardiomyopathy and renal abnormalities. Birth weight was 1487 g (-1.77 SD), length 42 cm (-1,14 SD) and OFC $28 \mathrm{~cm}$ (-2,19 SD). Post-natal investigations revealed a ventricular septal defect, which closed spontaneously, an atrial septal defect, multicystic left kidney, simplification of gyral pattern at brain MRI and gastroesophageal reflux which caused frequent vomiting. At our last examination (six years, she had moderate short stature $\left(107 \mathrm{~cm}, 5^{\circ}\right.$ centile) and a progressive microcephaly (OFC 42.5 cm, -5.2SD for statural age); no major dysmorphisms were detected. She had a mild intellectual disability. 


\section{GME_19 (ECEL1)}

This boy is the third child of a consanguineous couple (first cousins). One sister died at two months, she had cleft palate (no other information available); an elder brother is unaffected.

The proband presented with arthrogryposis (flexed knees, camptodactily, adducted thumbs, single palmar creases), bilateral ptosis, trismus, cleft palate, long philtrum, downturned corners of the mouth and intellectual disability. At 7 years he was $110 \mathrm{~cm}$ tall (-2,19 SD), weight was $14 \mathrm{~kg}$ (-2,29 SD) and OFC was $52 \mathrm{~cm}(+0,70 \mathrm{SD})$.

\section{GME_22 (COLEC11)}

The proband was the only child of consanguineous parents (first cousins) and presented with a cleft lip and submucosal cleft palate, together with a bone defect on both sides of the crista galli. He also had highly arched eyebrows, marked telecanthus, bifid nose, bilateral hearing loss, a small PDA and kidney malformation (horseshoe kidney with hypoplastic left kidney). Mild developmental delay was reported. At 7 years he was $104 \mathrm{~cm}$ tall (-3,31 SD), weight was $17 \mathrm{~kg}(+0,07 \mathrm{SD})$ and OFC was 50 cm (-0,42 SD). 
Supp Fig. 1

A

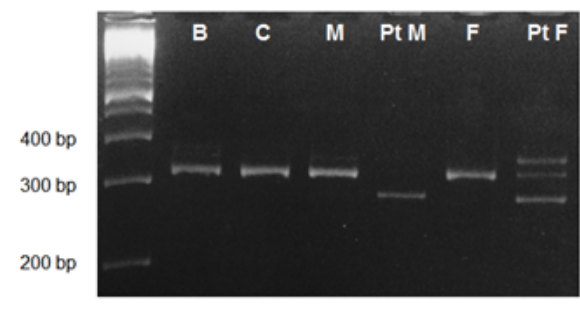

C

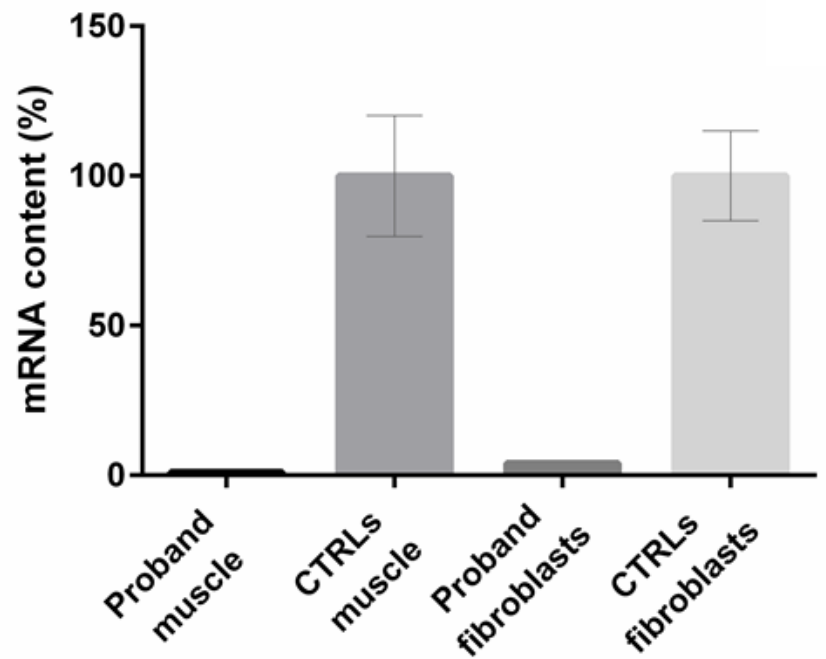

B

$\underset{\begin{array}{c}\text { CDNA } \\ \text { RefSeq }\end{array} \overbrace{\text { CAAAAGIGACAGATTAG AACATATGCTAAAAAA CAATTITTTAATICATTTCT }}^{\text {Exon } 8} \text { Exon } 9}{\text { Exon } 10}$
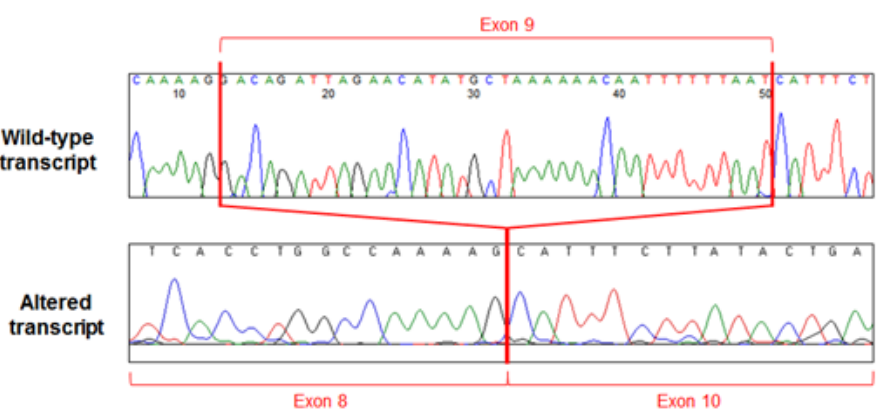
Supp Fig. 2
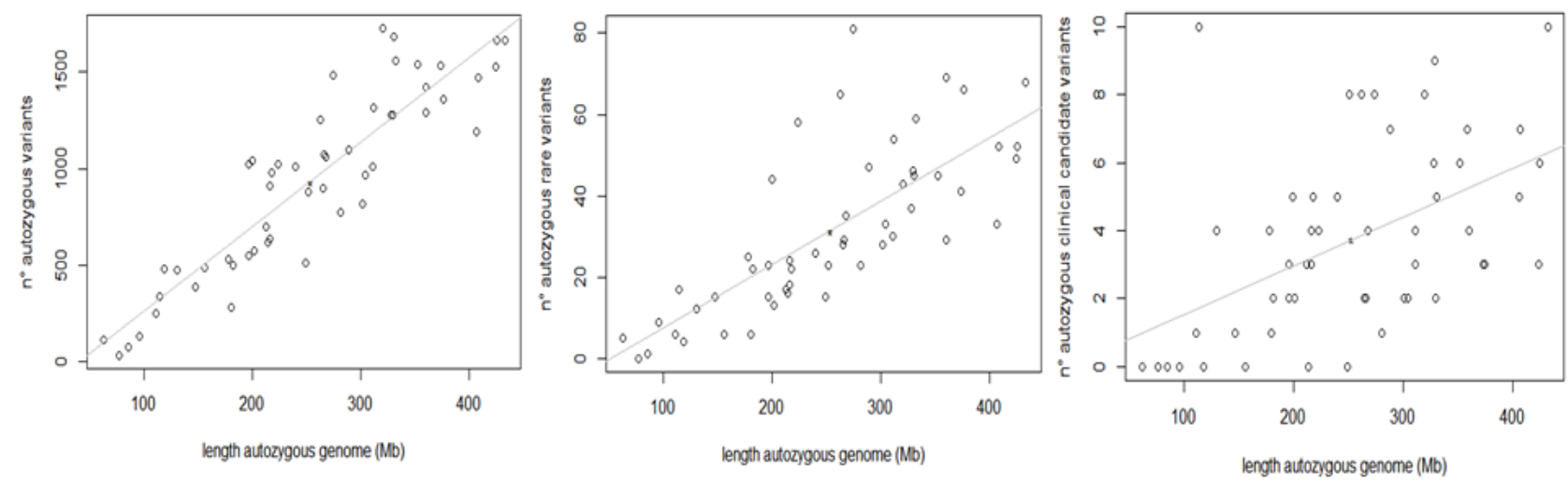

